\title{
An extensive study for correcting the nonlinear particle density measured by GRAPES-3 scintillator detectors
}

\section{A. Chandra, ${ }^{c}, *$ S. Ahmad, ${ }^{c}$ M. Chakraborty, ${ }^{a}$ S.R. Dugad, ${ }^{a}$ U.D. Goswami, ${ }^{l}$} S.K. Gupta, ${ }^{a}$ B. Hariharan, ${ }^{a}$ Y. Hayashi, ${ }^{b}$ P. Jagadeesan, ${ }^{a}$ A. Jain, ${ }^{a}$ P. Jain, ${ }^{d}$ S. Kawakami, ${ }^{b}$ H. Kojima, ${ }^{e}$ S. Mahapatra, ${ }^{i}$ P.K. Mohanty, ${ }^{a}$ R. Moharana,${ }^{j}$ Y. Muraki, ${ }^{g}$ P.K. Nayak, ${ }^{a}$ T. Nonaka, ${ }^{h}$ A. Oshima, ${ }^{e}$ B.P. Pant, ${ }^{j}$ D. Pattanaik, ${ }^{a, i}$ G.S. Pradhan, ${ }^{k}$ P.S. Rakshe, ${ }^{a}$ M. Rameez, ${ }^{a}$ K. Ramesh, ${ }^{a}$ L.V. Reddy, ${ }^{a}$ R. Sahoo, ${ }^{k}$ R. Scaria, ${ }^{k}$ S. Shibata, ${ }^{e}$ J. Soni, ${ }^{d}$ K. Tanaka, ${ }^{f}$ F. Varsi ${ }^{d}$ and M. Zuberi ${ }^{a}$ [The GRAPES-3 Collaboration]

${ }^{a}$ Tata Institute of Fundamental Research, Homi Bhabha Road, Mumbai 400005, India

${ }^{b}$ Graduate School of Science, Osaka City University, Osaka 558-8585, Japan

${ }^{c}$ Aligarh Muslim University, Aligarh 202002, India

${ }^{d}$ Indian Institute of Technology Kanpur, Kanpur 208016, India

${ }^{e}$ College of Engineering, Chubu University, Kasugai, Aichi 487-8501, Japan

${ }^{f}$ Graduate School of Information Sciences, Hiroshima City University, Hiroshima 731-3194, Japan

${ }^{g}$ Institute for Space-Earth Environmental Research, Nagoya University, Nagoya 464-8601, Japan

${ }^{h}$ Institute for Cosmic Ray Research, Tokyo University, Kashiwa, Chiba 277-8582, Japan

${ }^{i}$ Utkal University, Bhubaneshwar 751004, India

${ }^{j}$ Indian Institute of Technology Jodhpur, Jodhpur 342037, India

${ }^{k}$ Indian Institute of Technology Indore, Indore 453552, India

${ }^{l}$ Dibrugarh University, Dibrugarh 786004, India

E-mail: anuj.hep@gmail.com

The GRAPES-3 extensive air shower (EAS) array located at Ooty is equipped with 400 plastic scintillator detectors spread over an area of $25,000 \mathrm{~m}^{2}$ and a muon telescope of area $560 \mathrm{~m}^{2}$ built with 3,712 proportional counters. One of its principal objectives is to measure the primary cosmic ray energy spectrum in the TeV-PeV energy region. The response of the photo-multiplier tubes (PMTs) used in the plastic scintillator detectors becomes nonlinear at densities $>50$ particles- $\mathrm{m}^{-2}$ in large EAS. We describe a technique to correct for the nonlinearity of these PMTs, thereby extending the dynamic range of the detectors for observed particle densities up to $\sim 5000$ particles$\mathrm{m}^{-2}$. The details of the technique will be presented.

$37^{\text {th }}$ International Cosmic Ray Conference (ICRC 2021)

July 12 th - 23rd, 2021

Online - Berlin, Germany

${ }^{*}$ Presenter 


\section{Introduction}

High energy cosmic rays (CRs) are believed to be the messenger from the outer galaxies. The study of CRs may reveal the signatures about the occurrence of various phenomena as well as the nature of astrophysical objects, such as pulsars, supernovae explosions, active galactic nuclei and etc. Cosmic rays encounter complex magnetic fields in the inter-galactic, and extra-galactic space and get accelerated. The understanding of the nature of their sources and acceleration to the ultra-high energies (UHE) $E \geq 10^{15} \mathrm{eV}$ (PeV) are still eluding us although some progress is made at theoretical and experimental fronts [1]. The phenomena involved up to PeV energies can be explained with the help of shock acceleration of Fermi mechanism coupled with supernovae explosions. The higher energies CRs are modeled with the help of an extended version of same model with the concepts of shock progressing through stellar winds and acceleration through multiple shocks within the galactic disk. However, the power law energy spectrum of all-particle exhibits change in the spectral index from -2.7 to -3.0 at $\sim 3 \mathrm{PeV}$ and opens-up the debate for important checks for the models that explain the propagation and acceleration of CRs. The acceleration mechanism accompanies the change in the composition of CRs at around the knee energy and favors the scenario of supernova shock into stellar wind of a progenitor star at higher energies [2]. Therefore, the composition studies are sought to understand the phenomena occurring around the knee energies.

There are various investigations performed by direct [3-9] and indirect observations on primary $\mathrm{CRs}$ around the $\mathrm{PeV}$ energies. The continuous falling flux of primary CRs limits the detection of high energy CRs by the space based experiments. On the other hand, the detection of extensive air shower (EAS) by ground based experiments requires larger area and longer exposure time to understand the very high energy $\left(>10^{15} \mathrm{eV}\right)$ CRs. Some of the large area ground based experiments such as, CASA [10, 11], Tibet AS $\gamma$ [12], KASCADE [13, 14], MILAGRO [15] and ARGO-YBJ [16], have shown their measurements up to and above the knee energies.

The electrons, in an air shower is the most studied component for getting information on the shower, particularly for generating trigger, determining arrival direction and estimating the energy of the primary particle. A measurement of the lateral (radial) density distribution of particles in a shower using an array of unshielded charged particle detectors provides a good estimate of the observed number of particles in the shower,

$$
N_{e}^{o b s}=2 \pi \int_{r_{1}}^{r_{2}} r \rho_{e}(r) d r
$$

where, $\rho_{e}(r)$ is the density of particles at a distance $r$ from the core of the shower. With the core of the shower incident within the shower array, $r_{1}$ is generally taken to be 0 . The distance $r_{2}$ depends on the size of the shower array but the observed lateral distribution function is generally extrapolated to $r_{2}=\infty$ to obtain the total number $N_{e}^{t o t}$ of particles in the shower. Despite considerable fluctuations in the development of individual showers in the atmosphere and the differences in development profile for different primary nuclei, the shower size $\left(N_{e}^{o b s}\right.$ or $\left.N_{e}^{t o t}\right)$ is known to be a good parameter for estimating primary energy of showers.

Therefore, it is necessary to estimate the particle density precisely, and is required by any air shower experiments. The secondary particles produced in EAS is an increasing function of primary CRs energy [17]. In order to study the CRs of PeV energies the very precise estimation of secondary 
particles to be observed is utmost important. The Gamma Ray Astronomy at PeV Energies Phase-3 (GRAPES-3) array of plastic scintillator detectors is equipped with photo multiplier tubes (PMTs). While primaries of $\sim \mathrm{PeV}$ energies generate a large number of secondary particles in a shower and therefore, PMTs get saturated followed by the non-linear behavior of PMT in case of such large showers. The extension of the particle densities detected by detectors with the PMTs' linear behavious is already attempted [18] by using dual-PMT detectors. The particle densities observed by $\sim 75 \%$ of the detectors are still limited due to the constraint of PMTs non-linearity and saturation, and therefore an attempt is made to correct the observed non linearity observed up-to certain extent.

\section{GRAPES-3 Air Shower Array}

The GRAPES-3 (Gamma Ray Astronomy at PeV Energies Phase-3) experiment is assembled with a densely packed EAS array at Ooty in India $\left(11.4^{\circ} \mathrm{N}, 76.7^{\circ} \mathrm{E}, 2200 \mathrm{~m}\right.$ a.s.l). Currently, it is equipped with $\sim 400$ plastic scintillation detectors [2] each of $1 \mathrm{~m}^{2}$ area and the layout is shown in FIg.1. These shower detectors are laid in hexagonal structured array as a building block of a unit of 3-scintillator detectors making an equilateral triangle with $8 \mathrm{~m}$ inter detector separation. These plastic scintillation detectors are of two types:

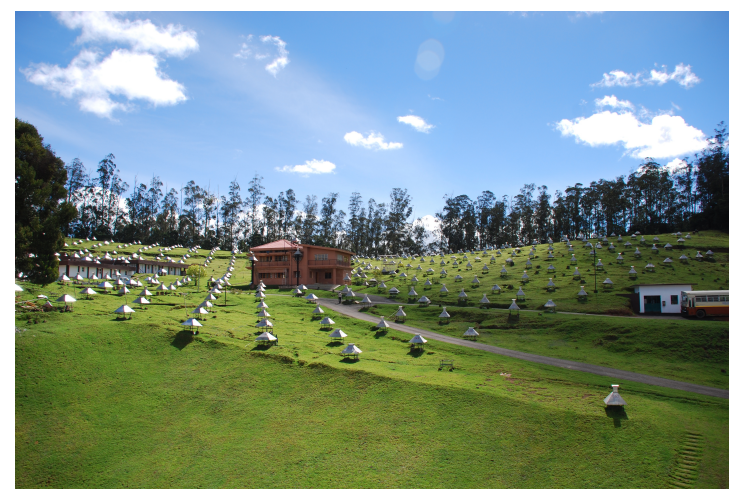

(a) Array Layout

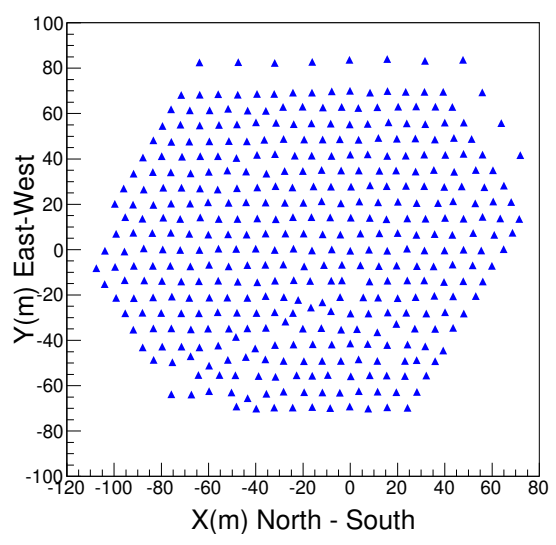

(b) Array Map

Figure 1: Plastic scintillation detectors deployed in hexagons rings over $25,000 \mathrm{~m}^{2}$ area.

- Cone Detectors: The cone type of scintillation detectors are the earliest configuration being used in the GRAPES-3 experiment [2]. All of them are of $1 \mathrm{~m}^{2}$ each and having four 50 $\times 50 \mathrm{~cm}^{2}$ area and $5 \mathrm{~cm}$ thick plastic scintallator blocks placed on the base of a trapezoidal shaped aluminum cone. A photo multiplier tube (PMT) ETL-9907SB02 of $5 \mathrm{~cm}$ diameter glass window is placed at $60 \mathrm{~cm}$ above the surface of scintillator blocks heading it's window towards scintillator blocks as shown in FIG.2 and other details are given in [2].

- Fiber Detectors: Due to the indirect detection of diffused photons from the wall of container in cone type detectors there are significant loss of signal, and consequently most of the cone type scintillator detectors have a photon output of $\leq 10$ photo-electrons[20]. On the other 


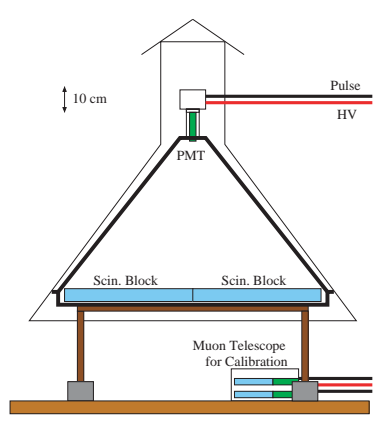

Figure 2: Schemetic of a shower detector, including the padels used for calibration.

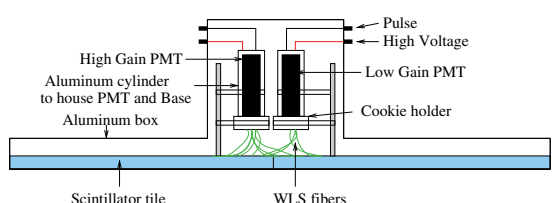

Figure 3: Cross sectional view of 2-PMT Fiber Detector.

hand, the geometry of the detector also resulted in a large spatial non-uniformity in the signal across the detector and a variation $\sim 30 \%$ is observed in photon output from the center to the edge of the detector. In order to overcome these limitations. we have developed the modified configuration of scintillation detectors by using wave-length shifting (WLS) fibers and the advantages of using WLS are mentioned in the work of P.K. Mohanty et al.[20]. The saturation of the PMTs encountered at very high particle densities in the earlier GRAPES-3 detectors, which limits their use over a wider range of primary CRs energies. To overcome the limitation of saturation of PMT, the WLS fiber detectors have been designed with two PMTs as shown in FIG.3. A dual-PMT detector is equipped with two PMTs, one is being operated at relatively higher operating voltage and hence called as HG-PMT and another one is operated at relatively lower operating voltage and hence can be used for obsering higher particle densities. With the advent feature of a dual-PMT detector, we achieve the observation of densities upto $\sim 10,000$ particles [18]. At present, GRAPES-3 is eqipped with 105 dual-PMT detectors out of 400 plastic scintillator detectors [18, 20].

\section{Methodology}

A method is eveloped in order to correct the observed non-linearity and saturated particle density of a high-gain(HG)-PMT. Previously, a similar approach was shown in [25], for correcting the non-linearity of the particle density observed by a HG-PMT with the help of extended density observed in dynamic range from a low-gain(LG)-PMT attached to the same double PMT detector. Here, we approach the saturation part of the observed density for the correction. The technique basically consists of using the spectral indices (slope) of density spectrum obtained from HG-PMT. We use this spectral indices value to obtain an extrapolated density spectrum. Equation below is used for the extrapolation of density spectrum by using the observed densities beyond 50 particles- $\mathrm{m}^{-2}$, spectral slope of density spectrum obtained in the range of particle density of $10-50$ particles $-\mathrm{m}^{-2}$, where,

$$
Y=A_{50} \cdot\left(\frac{X}{50}\right)^{-\gamma},
$$

$\mathrm{A}_{50}$ is the number of events $\gtrsim 50$ particles, $\gamma$ is the spectral indices obtained from the observed particle density spectrum by a HG-PMT of the same detector considered for correcting it's density, 
and $X$ is the number of particles $>50$ particles to be corrected from the region of non-linearity and saturation. Next, the corresponding integral number of events was computed using Eq.(2). This extrapolated spectrum along with the observed high-gain PMT particle density spectrum is shown in FIG.4.

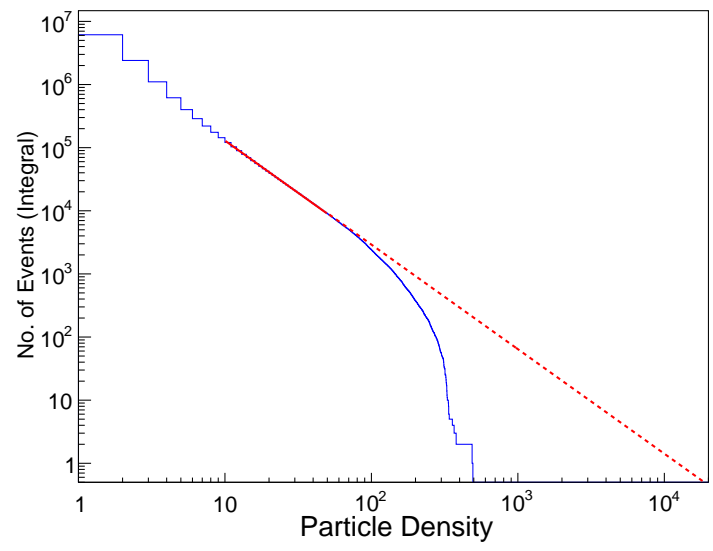

Figure 4: High-gain and extrapolated particle density spectrum.

A detailed method for correcting particle densities has been already discussed in [25] and a mathematical expression is also obtained in order to establish a relation between uncorrected and corrected particle densities, and can be explained by Eq.3. Where, A, B, and C are the free parameters and $X$ is the uncorrected particle density. This relation validates the correction up-to certain range of particle density, since afterwards the dependence of corrected particle density against uncorrected particle density shows very abrupt change, and, henceforth, does not follow the slowly varying exponential functionality. In addition, we try to improve the correction method by establishing a relation between corrected and uncorrected densities in the region of saturation. Here we try to correct the densities beyond the region of non-linearity by using a higher order polynomial.

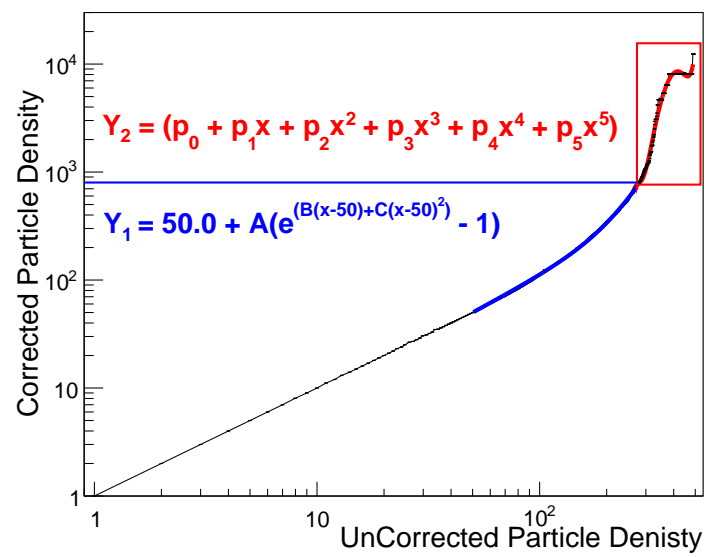

Figure 5: Dependence of corrected particle density on uncorrected particle density. 
The variation of the corrected particle density versus uncorrected particle density is shown in FIG.5. The errors are also introduced to each corrected particle density as a statistical error considered from the number of integral events from the distribution of uncorrected particle density.

$$
Y_{1}=50+A\left[\exp \left(B(X-50)+C(X-50)^{2}\right)-1\right]
$$

Where, $Y_{1}$ is the value of corrected particle density estimated empirically from Eq.3. The values of free parameters $\mathrm{A}, \mathrm{B}$ and $\mathrm{C}$ are evaluated after fitting the dependence of corrected particle density on uncorrected particle density in the region of non-linearity of the HG-PMT.

For correcting the density observed in the saturation region, a polynomial function of $5^{\text {th }}$ order is used to model the variation between corrected and uncorrected densities, and can be written as Eq.4. A clear fitting of the variation of corrected to uncorrected densities can be clearly seen in Fig.5 for the two regions.

$$
Y_{2}=p_{0}+p_{1} X+p_{2} X^{2}+p_{3} X^{3}+p_{4} X^{4}+p_{5} X^{5}
$$

As we obtain all the fitting parameters $\mathrm{A}, \mathrm{B}$, and $\mathrm{C}$ from Eq. 3 and $p_{0}, p_{1}, p_{2}, p_{3}, p_{4}$, and $p_{5}$ from Eq.4,

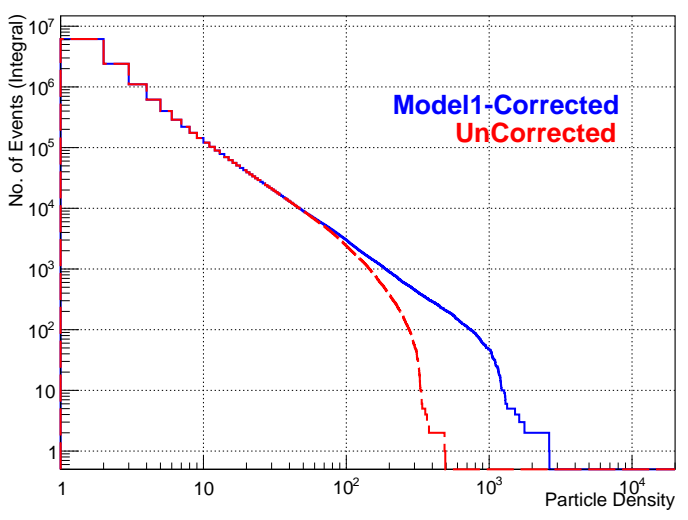

Figure 6: Correction of non-linearity region with slowly varying exponential function.

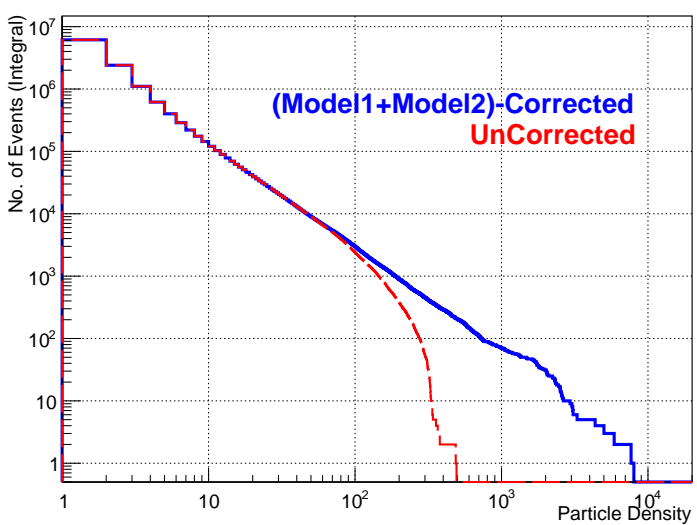

Figure 7: Correction of non-linearity and saturation region with the both functions.

we apply the correction method on event-by-event basis. Selection of the fitting model is decided on the basis of a limit considered on the ratio $\left(\mathrm{R}_{C U}\right)$ of corrected to uncorrected densities. Correction from Eq. 3 is valid only if the ratio $\mathrm{R}_{C U} \leq 2.76$, if higher the value of $\mathrm{R}_{C U}$ from 2.76 , brings the Eq.4 into consideration for further density correction in saturation region. The correction parameters are obtained after compiling one year (2017) of the data. Thereafter, correction of observed densities in non-linearity and saturation region are performed on event-by-event basis. For comparison purpose, integral particle density spectrum are obtained from observed densities and corrected densities from HG-PMT and are shown in Fig.6 and Fig.7.

\section{Results \& Discussion:}

A corrected integral density spectrum obtained by using Eq.3 is plotted and compared in FIg.6. The comparison between corrected and observed densities shows very much similar values upto 
$\sim(500-800)$ particles- $\mathrm{m}^{-2}$. On the other hand, Fig.7 shows a comparison in between corrected integral desnsity spectrum obtained from Eq. 3 and Eq.4 altogether corrected in the region of nonlinearity and saturation. The later corrected density spectrum shows extended particle densities in dynamic range. In addition, the extended correction correction can be validated by comparing with the observed density spectrum in the extended range by using LG-PMT attached with the same WLS-fiber dual-PMT detector [18] and the same is ahown in Fig.8. The extended density spectrum obtained from the observed LG-PMT and corrected from HG-PMT shows very similar values of particles $\sim 5000$ particles- $\mathrm{m}^{-2}$.

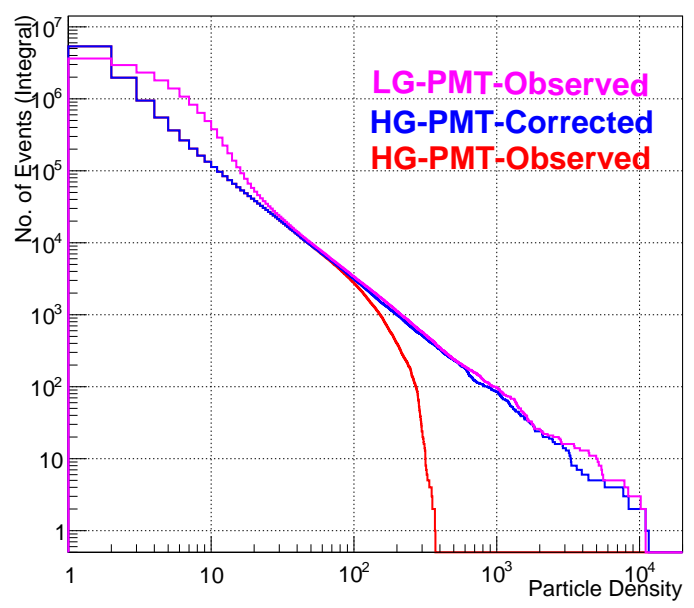

Figure 8: Corrected density is extended > 1000 particles and compared with density from LG-PMT.

\section{Conclusions}

The technique for the correction of non-linearity observed from HG-PMT is discussed and shows an extension in observed particle densities but limited upto $\sim(500-800)$ particles- $\mathrm{m}^{-2}$. As in the case of the latest method developed for correcting the saturation part of the observed HGPMT density, can be corrected upto the density $\sim 5000$ particles $-\mathrm{m}^{-2}$ and shows a good agreement with the observed particle densities in the extended range from LG-PMT. Both, the corrected and observed ranges shows very much similar values of number of events.

\section{Acknowledgement}

We thank D.B. Arjunan, G.P. Francis, V. Jeyakumar, S. Kingston, K. Manjunath, S. Murugapandian, S. Pandurangan, B. Rajesh, K. Ramadass, V. Santoshkumar, M.S. Shareef, C. Shobana, R. Sureshkumar and other colleagues for their help in running and maintenance of the GRAPES-3 experiment.

\section{References}

[1] V.S. Berezinskii, et al., Astrophysics of Cosmic Rays, North-Holland, Amsterdam, 1990. 
[2] S.K. Gupta et al., Nuclear Instruments and Methods in Physics Research A 540 (2005) 311-323.

[3] K. Asakimori, et al., Astrophys. J. 502 (1998) 278.

[4] M. Boezio et al., Astropart. Phys. 19 (2003) 583.

[5] Haino et al., Phys. Lett. B 594 (2004) 35.

[6] Y.S. Yoon et al., The Astrophys. J. 728 (2011) 122.

[7] M. Aguilar et al., Phys. Rev. Lett. 114, 171103 (2015).

[8] O. Adriani et al., Science 332 (2011) 69.

[9] J. Alcaraz et al., Phys. Lett. B 494 (2000) 193.

[10] D.J. Bird et al., 24 ${ }^{\text {th }}$ ICRC-1995, Vol. 2, August 28-September 8, 1995 in Rome, Italy. Edited by N. Iucci and E. Lamanna. International Union of Pure and Applied Physics, 1995., p.760.

[11] L. Fortson, Proceedings of the 26th ICRC-1999, August 17-25, 1999. Salt Lake City, Utah, USA. Under the auspices of the International Union of Pure and Applied Physics (IUPAP). Volume 3. Edited by D. Kieda, M. Salamon, and B. Dingus, p.125.

[12] M. Amenomori, et al., Nucl. Instr. and Meth. A 288 (1990) 619.

[13] T. Antoni, et al., Nucl. Instr. and Meth. A 513 (2003) 490;

T. Antoni, et al., Astropart. Phys. 14 (2001) 245.

[14] A. Haungs et al, Journal of Physics: Conference Series 409 (2013) 012005

[15] R. Atkins, et al., Nucl. Instr. and Meth. A 449 (2000) 478;

R. Atkins, et al., Astrophys. J. 595 (2003) 803.

[16] C. Bassi, et al., Nucl. Instr. and Meth. A 443 (2000) 342;

C. Bassi, et al., Astropart. Phys. 17 (2002) 151.

[17] A.A. Radu, et al., Romanian Reports in Physics, Vol. 60, No. 1, P. 45 - 55, 2008.

[18] A. Chandra et al., PoS (ICRC2017) 479.

[19] S.C. Tonwar, et al., Nuclear Physics B (Proc. Suppl.) 151 (2006) 477 - 480.

[20] P.K. Mohanty et al., Astroparticle Physics 31 (2009) 24 - 36.

[21] https : //www.hamamatsu.com/resources/pdf/etd/PMT_handbook_v3aE Chapter4.pdf

[22] X. Wang et al., Chinese Physics C 40, 8 (2016) 086003.

[23] PhototMultiplier Tubes Basics and Applications, $3^{\text {rd }}$ Edition, 2007, 160 - 161.

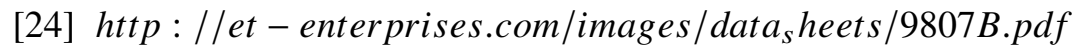

[25] A. Chandra, et al., PoS (ICRC2019) 216. 